\title{
A Compact Printed Filtering Antenna Using a Ground-Intruded Coupled Line Resonator
}

\author{
Chao-Tang Chuang and Shyh-Jong Chung, Senior Member, IEEE
}

\begin{abstract}
A compact printed filtering antenna with high bandedge gain selectivity is presented. Occupying about the same substrate area as a conventional antenna, the proposed structure not only serves as a radiator but also a second-order bandpass filter, with one filter pole provided by a $\Gamma$-shaped antenna and the other by a newly proposed coupled line resonator. High band-edge selectivity is achieved due to two additional stop-band transmission zeros provided by the coupled line resonator. To minimize the required area and reduce the spurious radiation, a coupled line structure composed of a microstrip line and a coplanar waveguide by broadside coupling is adopted and intruded into the $\Gamma$-shaped antenna area. According to the filter specifications, a design procedure for the proposed filtering antenna is depicted in detail. One example at $2.45 \mathrm{GHz}$ with a second-order Chebyshev bandpass filter of $0.1 \mathrm{~dB}$ equal-ripple response is tackled. As compared to the conventional $\Gamma$-shaped antenna, the proposed filtering antenna not only possesses a similar antenna gain but also provides better bandedge gain selectivity and flat passband gain response. The measured results, including the S-parameters, total radiated power, and antenna gains versus frequency, have good agreement with the designed ones.
\end{abstract}

Index Terms-Band-edge selectivity, bandpass filter, coupled line resonator, filtering antenna.

\section{INTRODUCTION}

W ITH the rapid development of wireless communication systems, the requirements for compact, low-cost, and low profile passive components are demanded in recent years. To achieve these purposes, various efforts could be done in a single circuit module. Integration of antenna and bandpass filter in one module is one of the ways to achieve miniaturization and improved performance of microwave front ends.

There have been numerous studies in the literature for integrating the filter and antenna into a single microwave device [1]-[4]. In order to reduce circuit area, a pre-design bandpass filter with suitable configuration was directly inserted into the feed position of a patch antenna [1]. Also to increase the bandwidth, the bandpass filter can be integrated properly with the antenna [2]-[4] by using an extra impedance transformation structure in between the filter and the antenna. Nevertheless, the tran-

Manuscript received November 01, 2010; revised February 15, 2011; accepted March 18, 2011. Date of publication August 04, 2011; date of current version October 05, 2011. This work was supported in part by the National Science Council, R.O.C., under Contract NSC 97-2221-E-009-041-MY3.

The authors are with the Institute of Communication Engineering, National Chiao Tung University, Hsinchu, Taiwan 30050, R.O.C. (e-mail: sjchung @ cm. nctu.edu.tw).

Color versions of one or more of the figures in this paper are available online at http://ieeexplore.iee.org.

Digital Object Identifier 10.1109/TAP.2011.2163777

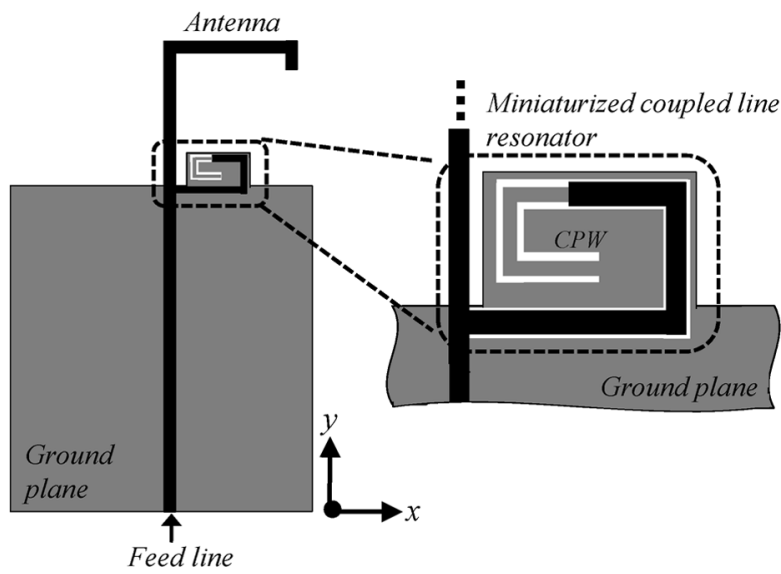

Fig. 1. The proposed compact second-order filtering antenna.

sition structure needs extra circuit area, and the designs did not give good filter responses in the frequency ranges.

In more recent years, the integration approach that the last resonator and the load impedance of the bandpass filter were substituted by an antenna, which is called the filtering antenna, has been discussed. The filtering antennas designed following the synthesis process of the bandpass filter have been presented in [5]-[8]. Although they have been done based on the co-design approach, these filtering antennas did not show good filter performance, especially the band-edge selectivity and stop-band rejection. This is due to the lack of the extraction of the antenna's equivalent circuit over a suitable bandwidth. Only that at the center frequency was extracted and used in the filter synthesis. Moreover, the total radiated powers and the antenna gains versus frequency, which are the important characteristic of the filtering antenna, were not examined in these studies.

In this study, a new compact printed filtering antenna, which is composed of a miniaturized coupled line resonator and a $\Gamma$-shaped antenna, as shown in Fig. 1, is proposed. The coupled line resonator in the filtering antenna can provide one pair of transmission zeros with tunable frequencies to achieve the purpose of the high band-edge selectivity. This paper is organized as follows: Section II illustrates the equivalent circuit and performance of the coupled line resonator; Section III shows the design procedure for the filtering antenna. A structure is synthesized as an example. The design and measurement of the proposed compact filtering antenna are then presented in Section IV, followed by a conclusion in Section V.

\section{ANALYSIS OF THE COUPLED LINE RESONATOR}

In the design of a narrow-band bandpass filter, one needs to use shunt $L C$ resonators with high capacitances. Although these 


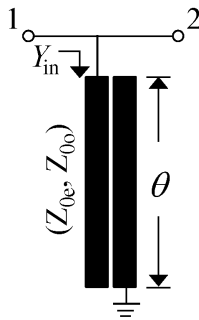

(a)

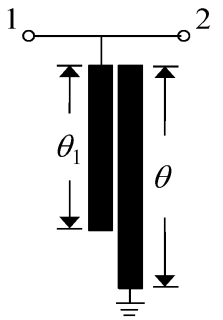

(b)
Fig. 2. Geometry of the coupled line resonators with (a) equal lengths and (b) unequal lengths.

high-capacitance $L C$ resonators can be implemented by using $\lambda / 4$ short-circuited microstrip stubs, it needs quite large circuit area because of the very wide microstrip line used. In [9], [10], the dual-behavior resonators are designed by associating two different parallel open-circuited stubs. Each stub brings its own transmission zero depending on its fundamental resonant condition. Even so, these resonators also suffer from large circuit area because of the use of the two open-circuited stubs. Recently, the authors proposed the coupled line resonators as shown in Fig. 2(a) [11] to solve this problem, which provides high equivalent capacitance while occupies a reasonable circuit area. In addition, without using the technique of cross-coupling between non-adjacent resonators [12], the proposed coupled line resonator itself produces one pair of tunable symmetric transmission zeros at the two sides of the passband, which greatly increase the band-edge selectivity. In this study, detail analyses of the coupled line resonator are presented. Also, an extended version with different lengths of the coupled lines as shown in Fig. 2(b) is introduced. As will be shown later, this new structure has the advantage of producing two asymmetric transmission zeros that may be contributive to the design flexibility.

The coupled line resonator with equal lengths shown in Fig. 2(a) is composed of a quarter-wavelength open-circuited stub and a quarter-wavelength short-circuited stub. These two resonant microstrip stubs couple with each other through the gap between them. Physically, the open-circuited stub is equivalent to a series $L C$ resonator, and the short-circuited stub a shunt $L C$ resonator. And the coupling gap, which mainly offers electrical coupling between the resonators, functions as an admittance inverter (or $J$-inverter) [13]. Therefore, the proposed structure can be equivalent to the circuit shown in Fig. 3(a). Note that due to the presence of the coupling, the two resonators $\left(L_{\mathrm{a}}, C_{\mathrm{a}}\right)$ and $\left(L_{\mathrm{b}}^{\prime}, C_{\mathrm{b}}^{\prime}\right)$ have different resonant frequencies, although the two coupled stubs are with equal lengths. This equivalent circuit can be further transformed into the circuit shown in Fig. 3(b), which consists of two series $L C$ resonators connected in parallel, with resonant frequencies at $f_{\mathrm{a}}$ and $f_{\mathrm{b}}$, respectively. It is thus evident that the proposed structure provides two transmission zeros at these two resonant frequencies.

We now set up the quantitative equivalence between the coupled line resonator (Fig. 2(a)) and the equivalent circuit (Fig. 3(b)) in the following three steps: First, derive the input admittances of the circuits; secondly, equate the two admittances and their derivatives, respectively, at the center frequency, and

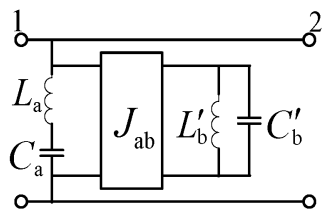

(a)

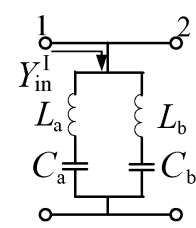

(b)

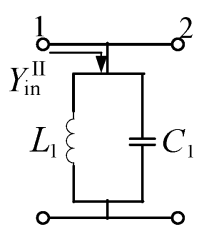

(c)
Fig. 3. (a) Equivalent circuit of the coupled line resonator with equal lengths. (b) The composite shunt resonator with two series $L C$ resonators in parallel. (c) The equivalent shunt $L C$ resonator around the resonant frequency $f_{\mathrm{r}}$.

also let the two circuits with the same transmission zeros; thirdly, extract the equivalent circuit components from the above-obtained equations.

In the first step, one first derives the admittances of these two circuits as [13]:

$$
\begin{aligned}
& Y_{\mathrm{in}}=-j \frac{\left(Z_{0 \mathrm{e}}+Z_{0 \mathrm{o}}\right) \sin 2 \theta}{\left(Z_{0 \mathrm{e}}-Z_{0 \mathrm{o}}\right)^{2}-\left(Z_{0 \mathrm{e}}+Z_{0 \mathrm{o}}\right)^{2} \cos ^{2} \theta} \\
& Y_{\mathrm{in}}^{\mathrm{I}}=-j\left[\frac{1}{2 \pi f L_{\mathrm{a}}\left(1-\frac{f_{\mathrm{a}}^{2}}{f^{2}}\right)}+\frac{1}{2 \pi f L_{\mathrm{b}}\left(1-\frac{f_{\mathrm{b}}^{2}}{f^{2}}\right)}\right]
\end{aligned}
$$

where $Y_{\text {in }}$ and $Y_{\text {in }}^{\mathrm{I}}$ are the admittances of the coupled line resonator and the equivalent circuit in Fig. 3(b), respectively. $Z_{0 \mathrm{e}}$ and $Z_{0 \mathrm{o}}$ are the even-mode and odd-mode impedances of the coupled line. $\theta$ is the electrical length, $\theta=\pi f / 2 f_{r}$, with $f_{\mathrm{r}}$ the corresponding resonant frequency. $f_{\mathrm{a}}=\left(2 \pi \sqrt{L_{\mathrm{a}} C_{\mathrm{a}}}\right)^{-1}$, $f_{\mathrm{b}}=\left(2 \pi \sqrt{L_{\mathrm{b}} C_{\mathrm{b}}}\right)^{-1}$.

Note that, like the equivalent $L C$ circuit, the coupled line resonator has a transmission pole $\left(Y_{\text {in }}=0\right)$ at $\theta=\pi / 2$ (or $f=f_{\mathrm{r}}$ ) and two transmission zeros $\left(Y_{\text {in }} \rightarrow \infty\right)$ at $\theta=\sin ^{-1}\left[2 \sqrt{Z_{0 \mathrm{e}} Z_{0 \mathrm{o}}} / Z_{0 \mathrm{e}}+Z_{0 \mathrm{o}}\right]$ and $\pi-\sin ^{-1}\left[2 \sqrt{Z_{0 \mathrm{e}} Z_{0 \mathrm{o}}} / Z_{0 \mathrm{e}}+Z_{0 \mathrm{o}}\right]$. It is noticed that the two zeros are symmetric to the transmission pole.

In the second step, the circuit shown in Fig. 3(b) is equivalent to the coupled line resonator by letting $Y_{\text {in }}\left(f_{\mathrm{r}}\right)=Y_{\mathrm{in}}^{\mathrm{I}}\left(f_{\mathrm{r}}\right)$, $\left.\left(d Y_{\mathrm{in}} / d f\right)\right|_{f_{\mathrm{r}}}=\left.\left(d Y_{\mathrm{in}}^{\mathrm{I}} / d f\right)\right|_{f_{\mathrm{r}}}$, and also making their transmission zeros the same. One then obtains the following four equations:

$$
\begin{gathered}
\frac{1}{2 \pi f_{\mathrm{r}} L_{\mathrm{a}}\left(1-\frac{f_{\mathrm{a}}^{2}}{f_{\mathrm{r}}^{2}}\right)}+\frac{1}{2 \pi f_{\mathrm{r}} L_{\mathrm{b}}\left(1-\frac{f_{\mathrm{b}}^{2}}{f_{\mathrm{r}}^{2}}\right)}=0 \\
\frac{1+\frac{f_{\mathrm{b}}^{2}}{f_{\mathrm{r}}^{2}}}{2 \pi f_{\mathrm{r}} L_{\mathrm{a}}\left(1-\frac{f_{\mathrm{a}}^{2}}{f_{\mathrm{r}}^{2}}\right)^{2}}+\frac{Z_{0 \mathrm{e}}+Z_{0 \mathrm{o}}}{2 \pi f_{\mathrm{r}} L_{\mathrm{b}}\left(1-\frac{f_{\mathrm{b}}^{2}}{f_{\mathrm{r}}^{2}}\right)^{2}}=\pi \frac{(2 \mathrm{a})}{\left(Z_{0 \mathrm{e}}-Z_{0 \mathrm{o}}\right)^{2}} \\
f_{\mathrm{a}}=\frac{2 f_{\mathrm{r}}}{\pi} \sin ^{-1}\left(\frac{2 \sqrt{Z_{0 \mathrm{e}} Z_{0 \mathrm{o}}}}{Z_{0 \mathrm{e}}+Z_{0 \mathrm{o}}}\right) \\
f_{\mathrm{b}}=2 f_{\mathrm{r}}-f_{\mathrm{a}} .
\end{gathered}
$$

Once the dimensions of the coupled line resonator are given, $f_{\mathrm{r}}, Z_{0 \mathrm{e}}$, and $Z_{0 \mathrm{o}}$ will be determined. In the last step, one can thus get the four circuit parameters $\left(L_{\mathrm{a}}, C_{\mathrm{a}}, L_{\mathrm{b}}, C_{\mathrm{b}}\right)$ in Fig. 3(b) by solving these four equations.

Furthermore, near the resonant frequency $f_{\mathrm{r}}$, the coupled line resonator can be approximated as a shunt $L C$ resonator, as shown in Fig. 3(c), since one of the series $L C$ resonators in 


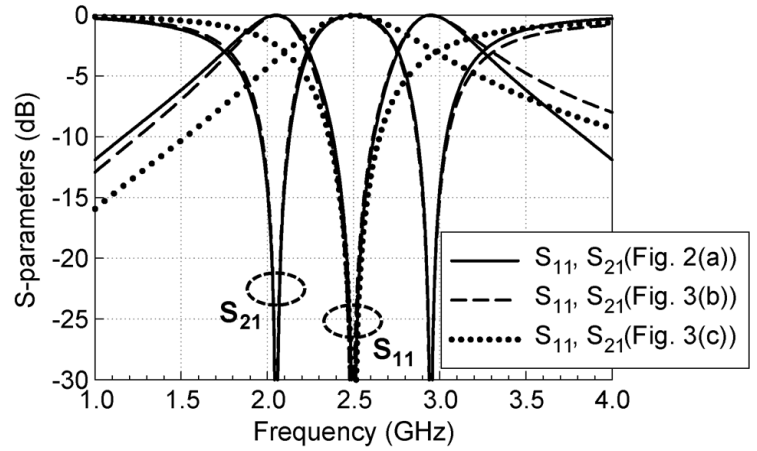

Fig. 4. Circuit simulation of the coupled line resonator with equal lengths in comparison with the ones of its corresponding equivalent circuits (Figs. 3(b) and (c)). The line width and gap size of the coupled line resonator are $0.5 \mathrm{~mm}$ and $0.2 \mathrm{~mm}$.

Fig. 3(b) shows inductive property and the other capacitive at the frequency in between $f_{\mathrm{a}}$ and $f_{\mathrm{b}}$. The equivalence between these two circuits are established by equalizing their resonant frequencies and the admittance derivatives, which yield

$$
f_{\mathrm{r}}=\frac{1}{2 \pi \sqrt{L_{1} C_{1}}}
$$

and

$$
\frac{\left(Z_{0 \mathrm{e}}+Z_{0 \mathrm{o}}\right)}{\left(Z_{0 \mathrm{e}}-Z_{0 \mathrm{o}}\right)^{2}}=\frac{2}{\pi} \sqrt{\frac{C_{1}}{L_{1}}} .
$$

The circuit components $L_{1}, C_{1}$ can thus be calculated while the impedances $\left(Z_{0 \mathrm{e}}, Z_{0 \mathrm{o}}\right)$ and $f_{\mathrm{r}}$ are given.

Fig. 4 shows the circuit simulation results by AWR [14] for a coupled line resonator with line width of $0.5 \mathrm{~mm}$ and gap 0.2 $\mathrm{mm}$, fabricated on a $0.508 \mathrm{~mm}$ thick Rogers 4003 substrate with a dielectric constant 3.38 and loss tangent of 0.0027 . Here, the ideal transmission lines for the coupled line resonator are considered. The electric length of the coupled lines is $\theta=90^{\circ}$, which corresponds to a resonant frequency at $2.5 \mathrm{GHz}$. To get the equivalent circuits, one first calculates the impedances $Z_{0 \mathrm{e}}$ and $Z_{0 \mathrm{o}}$ as $100 \Omega$ and $57 \Omega$ respectively. Then, based on (2c) and $(2 \mathrm{~d})$, the two transmission zeros, $f_{\mathrm{a}}$ and $f_{\mathrm{b}}$, are calculated to be $2.05 \mathrm{GHz}$ and $2.95 \mathrm{GHz}$. Finally, the circuit components in Figs. 3(b) and (c) can be obtained by using (2a), (2b), (3), and (4), which are $\left(L_{\mathrm{a}}, C_{\mathrm{a}}\right)=(8.46 \mathrm{nH}, 0.71 \mathrm{pF}),\left(L_{\mathrm{b}}, C_{\mathrm{b}}\right)=$ $(7.07 \mathrm{nH}, 0.41 \mathrm{pF})$, and $\left(L_{1}, C_{1}\right)=(0.49 \mathrm{nH}, 8.26 \mathrm{pF})$. It is seen from the figure that, the frequency responses of the equivalent circuit in Fig. 3(b) match very well with those of the coupled line resonator over the full frequency band. Not only with the same transmission pole and zeros, the curves are completely overlapped in the frequency range between $f_{\mathrm{a}}$ and $f_{\mathrm{b}}$. Also, the responses of the circuit in Fig. 3(c) agree well with the ones of the coupled line resonator near the resonant frequency $f_{\mathrm{r}}$.

As will be shown later, when designing a filtering antenna, the equivalent circuit components are first obtained based on the filter specifications, from which the structure dimensions have to be decided. To this end, one may use (2c) and (4) to derive the even- and odd-mode characteristic impedances of the coupled line resonator as:

$$
\begin{aligned}
& Z_{0 \mathrm{e}}=\frac{1}{2 k_{2}}\left[\frac{1}{1-k_{1}}+\frac{1}{\sqrt{1-k_{1}}}\right] \\
& Z_{0 \mathrm{o}}=\frac{1}{2 k_{2}}\left[\frac{1}{1-k_{1}}-\frac{1}{\sqrt{1-k_{1}}}\right]
\end{aligned}
$$

where $k_{1}=\sin ^{2}\left(\pi f_{\mathrm{a}} / 2 f_{\mathrm{r}}\right), k_{2}=(2 / \pi) \sqrt{C_{1} / L_{1}}=4 f_{r} C_{1}$.

These impedances together with the given resonant frequency $f_{\mathrm{r}}$ can then be used to determine the resonator's dimensions [13].

In many applications, the required transmission zeros of a bandpass filter are not symmetric to the passband. It is thus necessary to modify the proposed resonator structure shown in Fig. 2(a) that possesses symmetric zeros. One of the solutions is shown in Fig. 2(b), in which the short-circuited stub remains the same length, i.e., $\theta=\pi / 2$ at the resonant frequency, while the open-circuited stub has a length longer $\left(\theta_{1}>\pi / 2\right)$ or shorter $\left(\theta_{1}<\pi / 2\right)$. Fig. 5 shows the circuit simulation results for the coupled line resonators with different $\theta_{1}$. The structure parameters are the same as those in Fig. 4 except the length of the open-circuited stub. It is observed that, as $\theta_{1}$ is decreased or increased from $\pi / 2$, the resonant frequency remains the same but the two transmission zeros move toward the higher or lower frequencies, as shown in Figs. 5(a) and (b), respectively. Furthermore, for the reason that a smaller circuit area is usually required and the fact that the higher transmission zero moves much faster than the lower one, the case of shorter $\theta_{1}$ is chosen in this study. In application, one may first design a coupled line resonator with equal length to determine the resonant frequency $f_{\mathrm{r}}$ and the approximate the frequency of the lower transmission zero, and then adjust the frequency of the higher transmission zero to the desired one by tuning the open stub's length. Note that as depicted in Fig. 5(a), the characteristics of the coupled line resonator keep the same at frequencies near $f_{\mathrm{r}}$, which means that, when incorporating this resonator into a bandpass filter, the filter's passband performance will not change due to the adjustment of the higher transmission zero. Another solution, that the open-circuited stub has a length $\theta_{1}=\pi / 2$ while the short-circuited stub has a variable length can also provide asymmetric transmission zeros but would have different resonant frequency $f_{\mathrm{r}}$, which is thus not considered here.

\section{SYNTHESIS OF THE FILTERING ANTENNA}

In this section, the synthesis of a filtering antenna by using the coupled line resonator is to be presented. The proposed filtering antenna is constructed by directly connecting the coupled line resonator to a $\Gamma$-shaped antenna, as shown in Fig. 6(a). Since the antenna is a variety of a monopole antenna, it has a series $R L C$ equivalent circuit as shown in Fig. 6(b) [15]. Here, $L_{\mathrm{A}}$ and $C_{\mathrm{A}}$ express the equivalent inductance and capacitance, respectively, and $R_{\mathrm{A}}$ corresponds to the antenna radiation resistance. It is noted that an extra shunt capacitance $C_{\mathrm{g}}$ is incorporated in the equivalent circuit here so that the whole circuit would have the same impedance behavior as the antenna itself in a wider frequency range [11]. This parasitic capacitance comes from the 


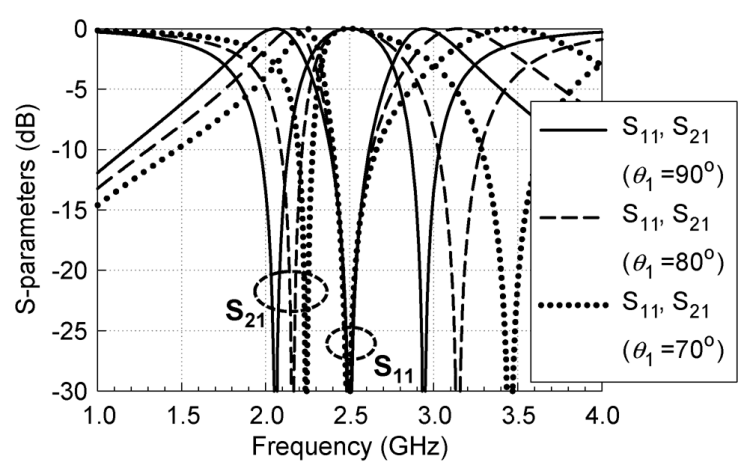

(a)

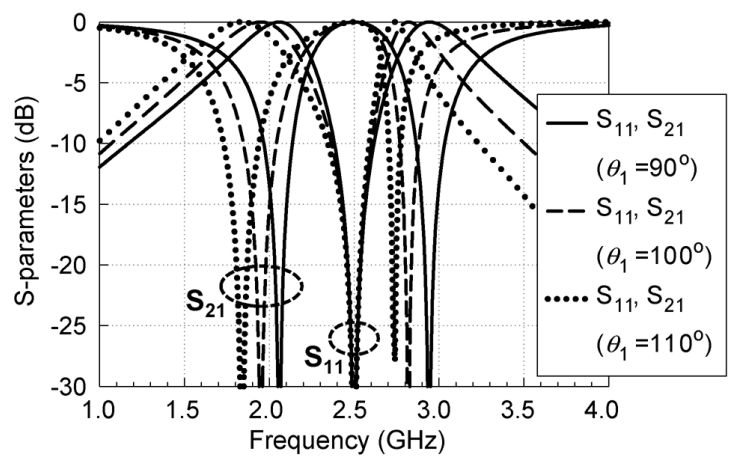

(b)

Fig. 5. Circuit simulation of the coupled line resonator with unequal lengths for different $\theta_{1}$. (a) $\theta_{1}$ decreases from $\pi / 2$. (b) $\theta_{1}$ increases from $\pi / 2$.

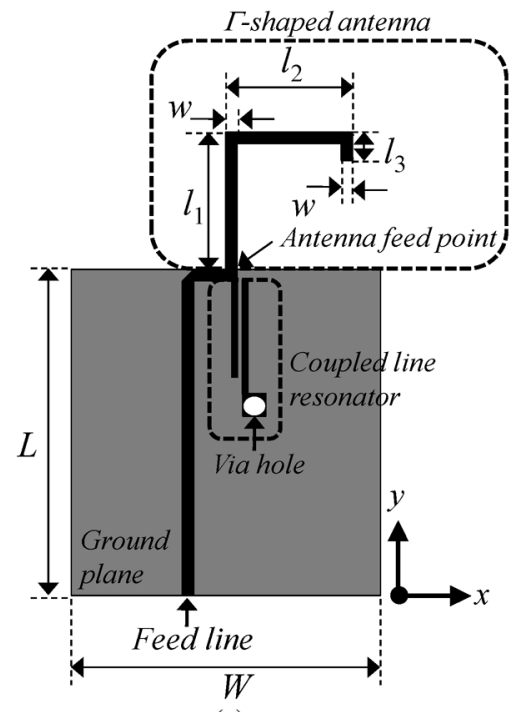

(a)

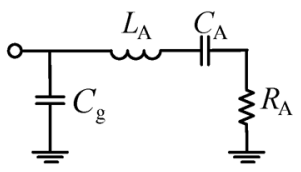

(b)
Fig. 6. (a) The geometry of the second-order filtering antenna. (b) The corresponding equivalent circuit of the $\Gamma$-shaped antenna.

accumulation of charges around the antenna feed point due to the truncation of the ground plane.

By using the equivalent circuits of the coupled line resonator and the $\Gamma$-shaped antenna, the second-order filtering antenna can be expressed by the equivalent circuit shown in Fig. 7(a). It can be observed that not only second-order response but also two extra transmission zeros near the filter's band-edge can be produced. Fig. 7(b) is a further equivalent circuit of the filtering

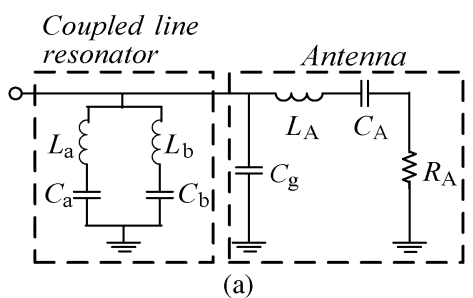

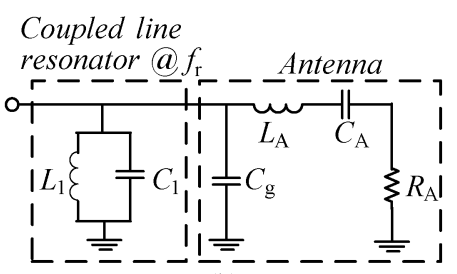

(b)

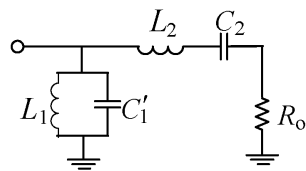

(c)
Fig. 7. (a) Equivalent circuit of the second-order filtering antenna. (b) Equivalent circuit of the filtering antenna while the resonant frequency of the coupled line resonator is near $f_{\mathrm{r}}$. (c) Equivalent circuit of the typical second-order bandpass filter.

antenna while the resonant frequency of the coupled line resonator is near $f_{\mathrm{r}}$. This circuit can then be transformed to a typical second-order bandpass filter as shown in Fig. 7(c), where $L_{2}=L_{\mathrm{A}}, C_{2}=C_{\mathrm{A}}, R_{0}=R_{\mathrm{A}}$, and $C_{1}^{\prime}=C_{1}+C_{\mathrm{g}}$. Note that due to the existence of the small capacitance $C_{\mathrm{g}}$, the required resonant frequency $f_{r}\left(=\left(2 \pi \sqrt{L_{1} C_{1}}\right)^{-1}\right)$ of the coupled line resonator is slightly larger than the operating frequency $f_{0}\left(=\left(2 \pi \sqrt{L_{1} C_{1}^{\prime}}\right)^{-1}=\left(2 \pi \sqrt{L_{2} C_{2}}\right)^{-1}\right)$ of the bandpass filter.

The design procedures of the proposed filtering antenna are listed below.

1) Specify the requirements of the bandpass filter to be synthesized, including the operating frequency $f_{0}$, the frequencies of the transmission zeros $\left(f_{\mathrm{a}}\right.$ and $\left.f_{\mathrm{b}}\right)$, the fractional bandwidth $\Delta$, and the type of the filter (e.g., bandpass filter with equal ripple). Therefore, the circuit elements $\left(L_{2}, C_{2}, R_{0}, L_{1}, C_{1}^{\prime}\right)$ of the typical bandpass filter in Fig. 7(c) can be calculated [13].

2) Choose the suitable dimensions of the $\Gamma$-shaped antenna and then extract the circuit elements $\left(L_{\mathrm{A}}, C_{\mathrm{A}}, R_{\mathrm{A}}, C_{\mathrm{g}}\right)$ of its equivalent circuit [11]. These circuit elements are used to function as the last (second) stage of the filter. Therefore, the antenna dimensions should be determined so as to satisfy the following requirements: $L_{\mathrm{A}}=L_{2}, C_{\mathrm{A}}=C_{2}$, and $R_{\mathrm{A}}=R_{0}$.

3) Use the component values of $L_{1}, C_{1}\left(=C_{1}^{\prime}-C_{\mathrm{g}}\right)$ obtained in the above procedures, together with the required resonant frequencies $f_{\mathrm{r}}\left(=\left(2 \pi \sqrt{L_{1} C_{1}}\right)^{-1}\right)$ and lower transmission zero $f_{\mathrm{a}}$, the characteristic impedances $\left(Z_{0 \mathrm{e}}, Z_{0 \mathrm{o}}\right)$ of the coupled line resonator with equal lengths can be calculated by using (5), and then its corresponding dimensions can be obtained.

4) Properly shorten the open stub's length $\left(\theta_{1}\right)$ in the coupled line resonator with unequal lengths so as to adjust the higher transmission zero to the required one $\left(f_{\mathrm{b}}\right)$. Note that although the position of the lower transmission zero is changed in this step, the variation is quite limited as shown in Fig. 5(a). If a more precise lower transmission zero is required, the frequency $f_{\mathrm{a}}$ used in the above procedure can 
be set a little smaller than the required one to compensate the effect when shortening the stub's length.

As an example, a second-order Chebyshev bandpass filter with a $0.1 \mathrm{~dB}$ equal-ripple response is tackled. The filtering antenna is designed on a $0.508 \mathrm{~mm}$ thick Rogers 4003 substrate with a dielectric constant of 3.38 and loss tangent of 0.0027 . The ground plane size $L \times W=50 \mathrm{~mm} \times 50 \mathrm{~mm}$. In the design, the full-wave simulation solver HFSS [16] is used for fine tuning in the last procedure. According to the design procedure, the specifications of the filter should be firstly chosen. Here, the bandpass filter has a operating frequency $f_{0}=2.45 \mathrm{GHz}$, a fractional bandwidth $\Delta=14 \%$, and $Z_{0}=50 \Omega$. The lower transmission zero $f_{\mathrm{a}}$ is set at $2 \mathrm{GHz}$, and the higher transmission zero $f_{\mathrm{b}}$ is $3.3 \mathrm{GHz}$. Based on these requirements, the circuit components of the filter can be calculated, which are $L_{1}=0.54 \mathrm{nH}$, $C_{1}^{\prime}=7.82 \mathrm{pF}, L_{2}=14.43 \mathrm{nH}, C_{2}=0.29 \mathrm{pF}$, and $R_{0}=37 \Omega$. Secondly, we have to determine the antenna sizes. After numerous simulations, we choose the dimensions of the $\Gamma$-shaped antenna as $l_{1}=15.2 \mathrm{~mm}, l_{2}=11.5 \mathrm{~mm}, l_{3}=3.1 \mathrm{~mm}$, and $w=1.17 \mathrm{~mm}$, which correspond to a set of extracted component values (i.e., $L_{\mathrm{A}}=14.4 \mathrm{nH}, C_{\mathrm{A}}=0.29 \mathrm{pF}, R_{\mathrm{A}}=37 \Omega$, $C_{\mathrm{g}}=0.37 \mathrm{pF}$ ) nearest to required one.

Thirdly, the circuit component $C_{1}$ and the resonant frequency $f_{\mathrm{r}}$ of the coupled line resonator are calculated, which are 7.45 $\mathrm{pF}$ and $2.51 \mathrm{GHz}$, respectively. The characteristic impedances $\left(Z_{0 \mathrm{e}}, Z_{0 \mathrm{o}}\right)=(89 \Omega, 47 \Omega)$ can then be gotten by using (5) and the given lower transmission zero, with which the dimensions of the coupled line resonator of equal lengths are obtained. The resultant line width, gap size, and length of the coupled line are $0.64 \mathrm{~mm}, 0.15 \mathrm{~mm}$, and $18.9 \mathrm{~mm}$, respectively. Finally, to reach a transmission zero at $3.3 \mathrm{GHz}$, the electric length $\theta_{1}$ of the open-circuited stub is shortened from $90^{\circ}$ to $70^{\circ}$ by simulation. To this end, all the structure dimensions have been determined. And the filtering antenna can be implemented by connecting the coupled line resonator and the $\Gamma$-shaped antenna, as shown in Fig. 6(a).

Fig. 8(a) shows the full-wave simulated return loss of the designed filtering antenna. The simulated result of the $\Gamma$-shaped antenna only is also shown for reference. The proposed filtering antenna exhibits a return loss larger than $15 \mathrm{~dB}$ over the passband, and provides better band-edge selectivity as compared to the $\Gamma$-shaped antenna only. Also observe that, with the center frequency at $2.45 \mathrm{GHz}$, the filtering antenna possesses two transmission poles at $2.33 \mathrm{GHz}$ and $2.57 \mathrm{GHz}$, which are caused from the repel of resonant frequencies due to mutual coupling between the coupled line resonator and $\Gamma$-shaped antenna [17]. Although not shown here, it has been found that the lower and higher transmission poles can be tuned independently by the coupled line resonator and the antenna, respectively.

Fig. 8(b) depicts the impedance behavior on the Smith chart of the filtering antenna, and Fig. 8(c) illustrates the current distributions at the center frequencies and the two transmission poles. At these three in-band frequencies, as observed from Fig. 8(b), the input impedance possesses negligible reactance but has similar resistances around $30 \Omega$. Due to the high radiation efficiency in the passband, the input resistance of the proposed antenna is nearly equal to the radiation resistance. Consequently, the currents distributed on the $\Gamma$-shaped antenna

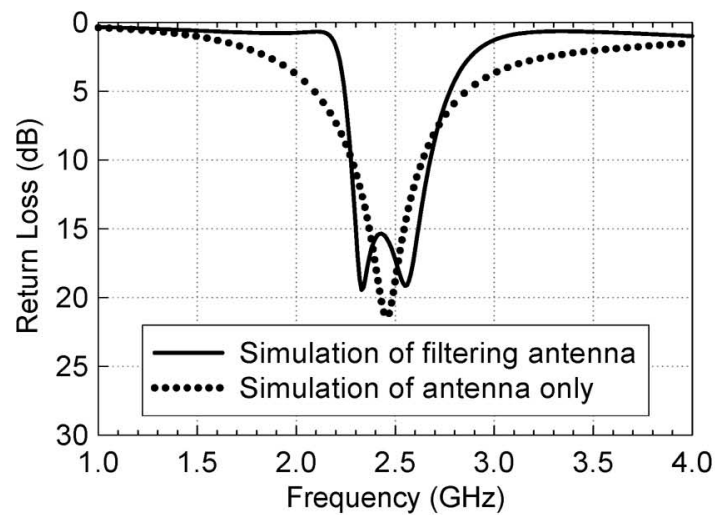

(a)

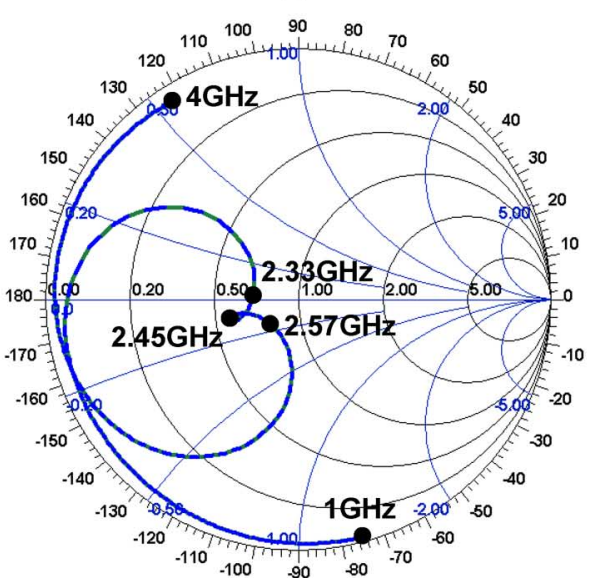

(b)

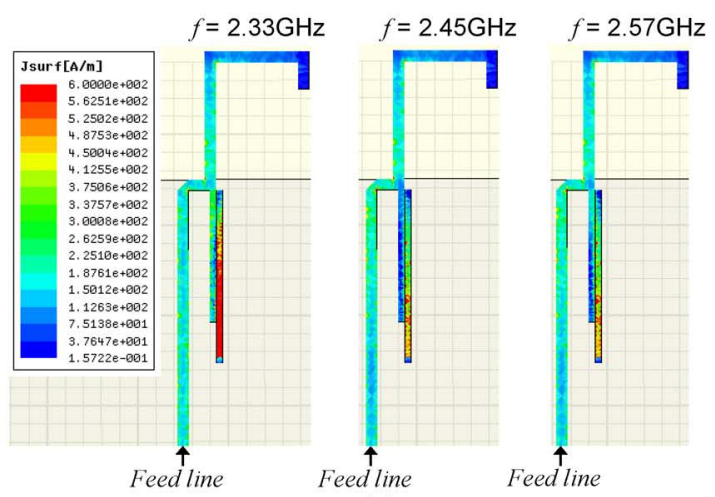

(c)

Fig. 8. (a) The full-wave simulated return loss of the filtering antenna in comparison with the simulated one of the $\Gamma$-shaped antenna. (b) The impedance behavior on the Smith chart of the filtering antenna. (c) The simulated current distributions of the filtering antenna at the center frequency and two transmission poles.

have about the same level at these frequencies, as can be observed from Fig. 8(c). Also notice from Fig. 8(c) that the current on the coupled line resonator at $2.33 \mathrm{GHz}$ is relatively large as compared to those at $2.45 \mathrm{GHz}$ and $2.57 \mathrm{GHz}$, which is apparent since the coupled line resonator is the main contributor for the lower transmission pole at $2.33 \mathrm{GHz}$.

Fig. 9 shows the full-wave simulated total radiated power of the filtering antenna in comparison with the simulated one of the $\Gamma$-shaped antenna. Here, the total radiated power has been normalized to the input power. It is seen that the filtering antenna exhibits a constant radiation power over the required frequency 


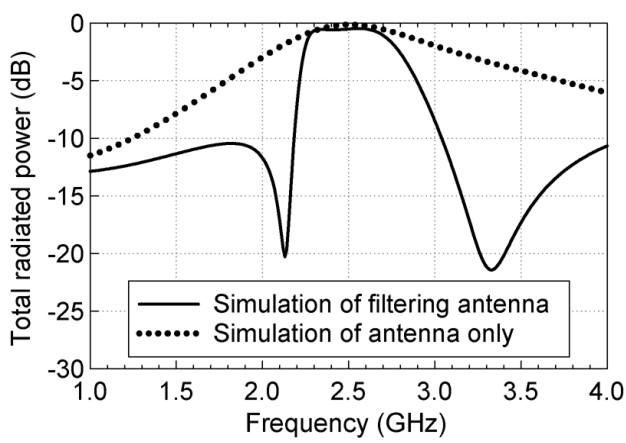

Fig. 9. The full-wave simulated total radiated power of the filtering antenna in comparison with the simulated ones of the $\Gamma$-shaped antenna.

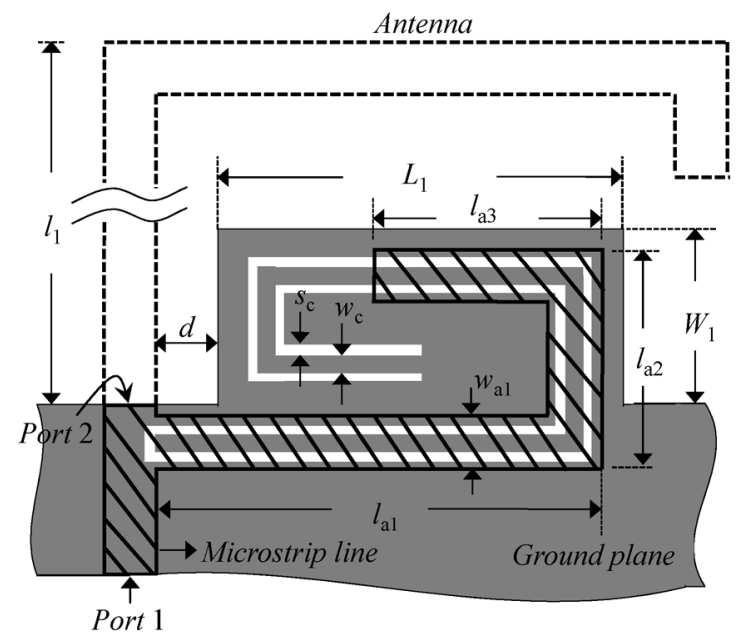

Fig. 10. Geometry of the proposed miniaturized coupled line resonator.

bandwidth, and has two radiation nulls at $2.13 \mathrm{GHz}$ and 3.33 $\mathrm{GHz}$ which are close to the design ones. Also, the proposed filtering antenna has much better stop-band suppression and band-edge selectivity. The corresponding radiation efficiency of the filtering antenna is about $88 \%$ in the passband, but is greatly reduced to $0.9 \%$ and $0.7 \%$ at the frequencies of the two radiation nulls. The input power is mostly reflected back due to the low return losses (about $0.7 \mathrm{~dB}$ ) at these two nulls.

\section{Compact Second-Order Filtering Antenna}

Although the proposed filtering antenna shown in Fig. 6(a) exhibits good radiation and filtering performances, it still occupies extra circuit area due to the use of the coupled line resonator. To realize a compact filtering antenna, a miniaturized coupled line resonator composed of a microstrip line open-circuited stub and a coplanar waveguide short-circuited stub by broadside coupling is proposed, as shown in Fig. 10, to intrude into the $\Gamma$-shaped antenna area. Herein, an extended ground plane $\left(W_{1} \times L_{1}\right)$ is employed for designing the folded coupled line resonator.

Fig. 11 shows the full-wave simulated scattering parameters for different dimensions of the miniaturized coupled line resonator. Note that the simulation result does not include the effect of the $\Gamma$-shaped antenna. It considers only the two-port folded coupled line resonator between ports 1 and 2 (see Fig. 10). The size $\left(W_{1} \times L_{1}\right)$ of the extended ground plane is $3.4 \times 7.5 \mathrm{~mm}^{2}$

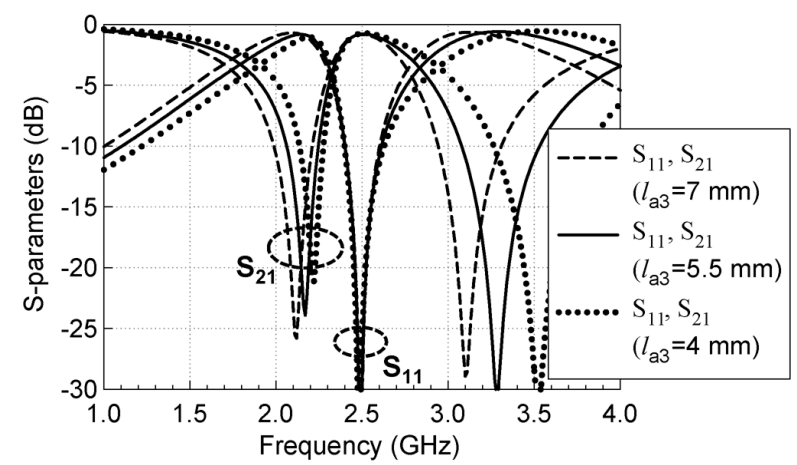

(a)

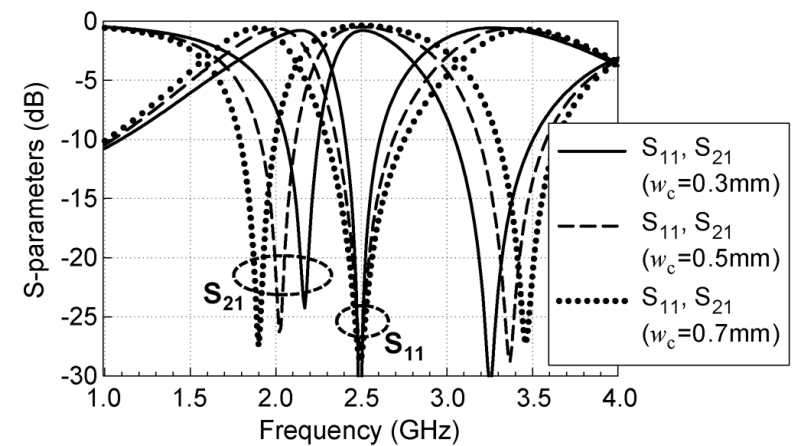

(b)

Fig. 11. Full-wave simulated S-parameters of the miniaturized coupled line resonator with unequal lengths. (a) different $l_{\mathrm{a} 3}$ with $w_{\mathrm{c}}=0.3 \mathrm{~mm}$. (b) different $w_{\mathrm{c}}$ with $l_{\mathrm{a} 3}=5.5 \mathrm{~mm}$. $\left(w_{\mathrm{a} 1}=1.2 \mathrm{~mm}, S_{\mathrm{c}}=0.2 \mathrm{~mm}\right.$ and $f_{\mathrm{r}}=$ $2.5 \mathrm{GHz}$ ).

and the distance $d$ between the extended ground plane and vertical strip line $\left(l_{1}\right)$ of the $\Gamma$-shaped antenna is $1 \mathrm{~mm}$. Fig. 11(a) depicts the effect of the length $l_{\mathrm{a} 3}$ of the microstrip line open-circuited stub. The coplanar waveguide short-circuited stub has a fixed total length of about a quarter wavelength and a line width $w_{\mathrm{c}}=0.3 \mathrm{~mm}$ and gap $s_{\mathrm{c}}=0.2 \mathrm{~mm}$. As shown, the miniaturized resonator has the same properties as the original one (Fig. 2(b)). It possesses a transmission pole and two side transmission zeros. Also, when the open-circuited stub is shortened (i.e., decreasing $l_{\mathrm{a} 3}$ ), the behavior near the center frequency is unchanged, and the two transmission zeros move toward the higher frequencies, with the higher transmission zero moving faster than the lower one.

Fig. 11(b) illustrates the effect of the line width $w_{\mathrm{c}}$ of the coplanar waveguide stub. As $w_{\mathrm{c}}$ increases, the center frequency remains the same due to the same short-circuited stub length $(\lambda / 4)$. While the two transmission zeros move away from the center frequency. This is because the increase of $w_{c}$ causes a stronger mutual coupling between the microstrip line and the coplanar waveguide, which thus results in a larger ratio of $Z_{0 \mathrm{e}} / Z_{0 \mathrm{o}}$. Therefore, from (2c) and (2d), the lower transmission zero appears at a lower frequency and the higher zero at a higher frequency.

In summary, the intruded miniaturized coupled line resonator follows the behaviors depicted in Section II. Thus, the design methodology described in the previous section can be applied directly to the proposed compact filtering antenna (Fig. 1). In the design, the filter specifications to be achieved and the dimensions of the $\Gamma$-shaped antenna are set the same as those in 
TABLE I

Dimensions of the Miniaturized COUPLED LiNE RESONATOR With UneQual LENGTHS IN THE COMPACT FILTERING ANTENNA

\begin{tabular}{|c|c|c|c|c|c|}
\hline$w_{\mathrm{a} 1}$ & $l_{\mathrm{a} 1}$ & $l_{\mathrm{a} 2}$ & $l_{\mathrm{a} 3}$ & $w_{\mathrm{c}}$ & $s_{\mathrm{c}}$ \\
\hline 1.2 & 8.3 & 4.4 & 5.5 & 0.3 & 0.2 \\
\hline
\end{tabular}

Unit: $\mathrm{mm}$

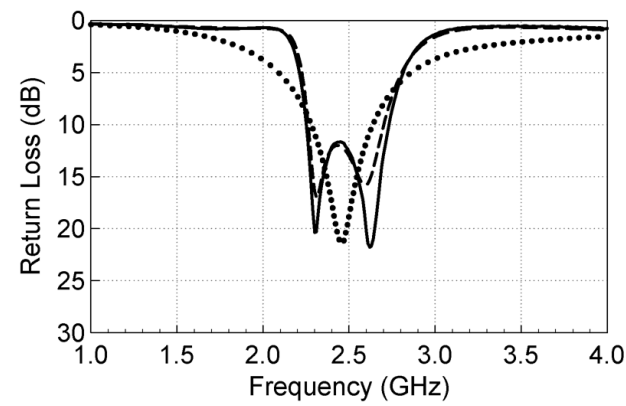

(a)

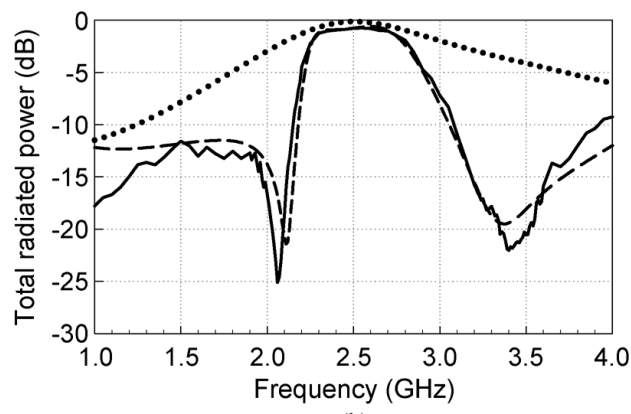

(b)

Fig. 12. The simulated and measured results of the compact filtering antenna in comparison with the simulated ones of the $\Gamma$-shaped antenna. (a) Return losses and (b) total radiated powers. [ - : measurement of compact filtering antenna; -.-. : simulation of compact filtering antenna; . . . . simulation of antenna only.]

Section III. And the final dimensions of the miniaturized coupled line resonator are listed in Table I. Fig. 12(a) and (b) shows the full-wave simulated and measured return losses and total radiated powers, respectively, of the compact filtering antenna. The simulated results of the $\Gamma$-shaped antenna are also shown for reference. It is seen that the full-wave simulated results of the compact filtering antenna are almost the same as those of the original design (Fig. 6(a)), which show two filter poles at 2.3 $\mathrm{GHz}$ and $2.6 \mathrm{GHz}$ and two radiation nulls at $2.11 \mathrm{GHz}$ and 3.31 $\mathrm{GHz}$. The simulated radiation efficiency at operating frequency $f_{0}=2.45$ is about $82 \%$, slightly lower than the original design, and those at the two transmission zeros are $0.7 \%$ and $1.1 \%$. The measured results agree quite well with the simulations. It is evident that, without suffering from the need of extra circuit area, the proposed compact filtering antenna shown in Fig. 1 has a flat passband radiation power as a function of frequency, high band-edge selectivity, and good stop-band suppression.

Figs. 13(a) and (b) show the full-wave simulated and measured antenna gains in the $+z$ and $+x$ directions versus frequency for the compact filtering antenna. The simulated antenna gains of the $\Gamma$-shaped antenna in these two directions are also shown for reference. As expected, the antenna gains for the compact filtering antenna are flat in the passband. Also, two clear radiation nulls in the stop band can be observed in the

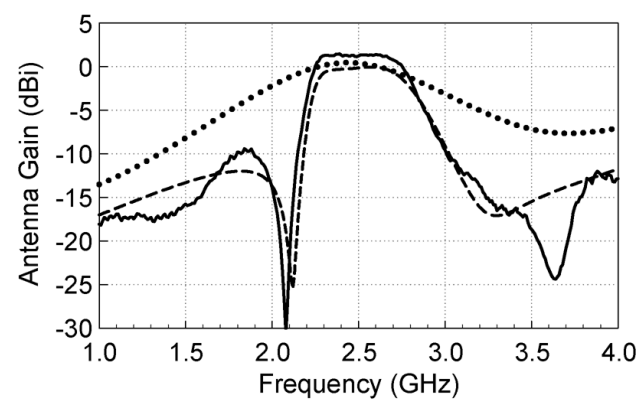

(a)

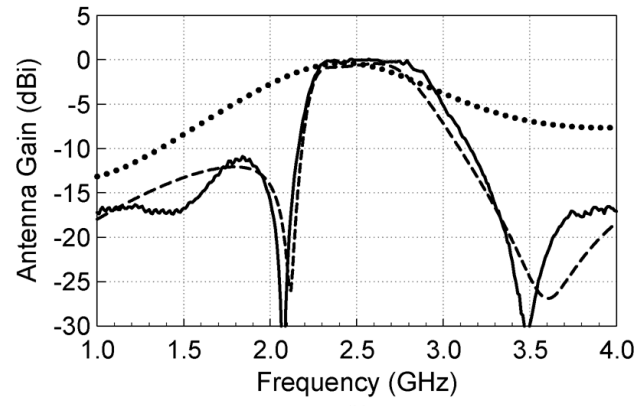

(b)

Fig. 13. The simulated and measured antenna gains versus frequency of the compact filtering antenna in comparison with the simulated ones of the $\Gamma$-shaped antenna. (a) In the $+z$ direction and (b) in the $+x$ direction. [ - : measurement of compact filtering antenna; ----: simulation of compact filtering antenna; . . . . : simulation of antenna only.]
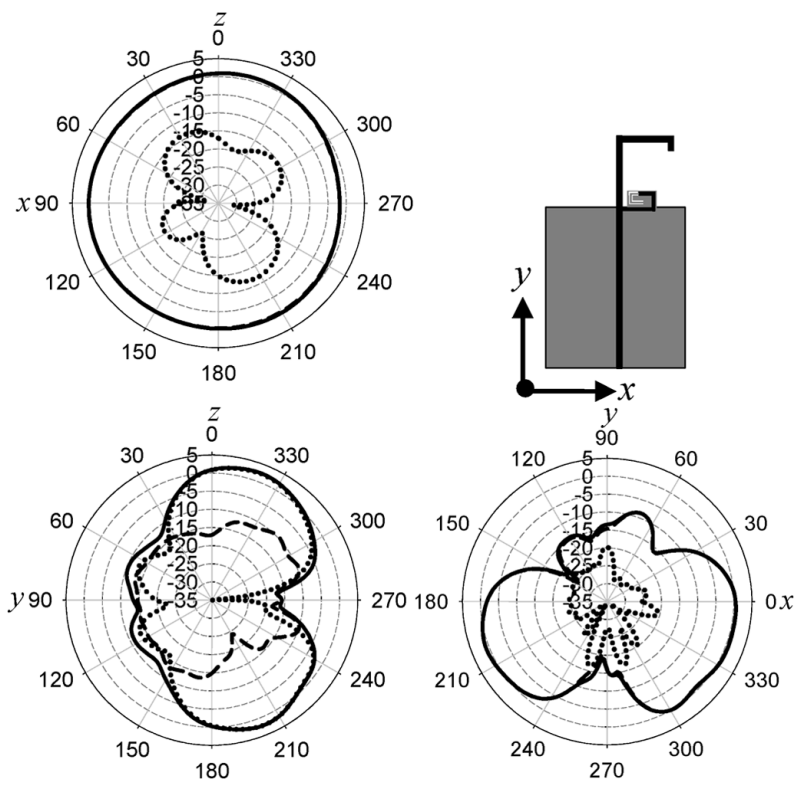

Fig. 14. Measured radiation patterns in the $x z, y z$, and $x y$ planes for the compact filtering antenna. $\left[--: E_{\text {total }} ;-: E_{\phi} ; \ldots: E_{\theta}\right] . f_{0}=2.45 \mathrm{GHz}$.

simulation results, which make the band-edge selectivity and stop-band suppression better than those of the reference structure. The first null of the antenna gain locates at a frequency exactly equal to that $(2.11 \mathrm{GHz})$ of the first null in the total radiation power response (Fig. 12(b)). While the frequency location of the second null deviates a little from that in Fig. 12(b) and depends on the observation angle, due to the influence of the antenna's radiation pattern. The measurement matches well to the simulation. The ripples in the stop band probably come from the 


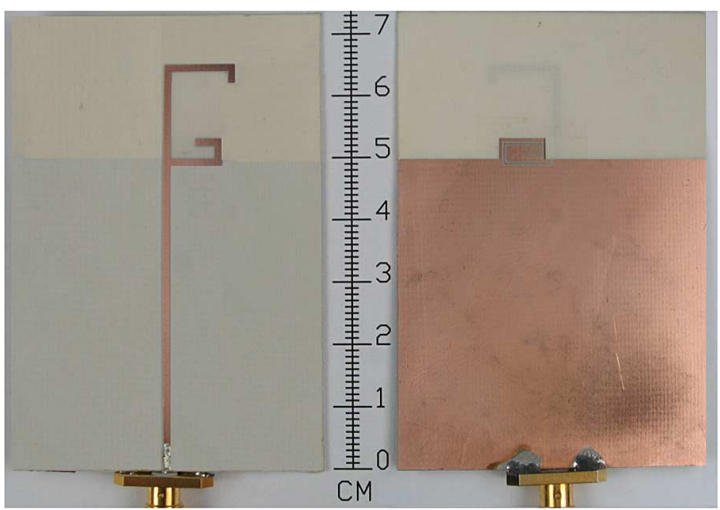

Fig. 15. Photograph of the compact filtering antenna. (left-side: top view; rightside: bottom view)

non-ideal anechoic chamber condition. The measured antenna gains at $f_{0}=2.45 \mathrm{GHz}$ in the $+z$ and $+x$ directions, including the circuitry loss, are $1.03 \mathrm{dBi}$ and $-0.18 \mathrm{dBi}$, respectively.

Fig. 14 depicts the measured radiation patterns of the compact filtering antenna at $f_{0}=2.45 \mathrm{GHz}$ in the three principal planes. The radiation pattern in the $x z$-plane is nearly omni-directional with peak gain of $1.2 \mathrm{dBi}$. Although not shown here, these patterns are about the same as the simulations and the measured results of the $\Gamma$-shaped antenna alone. Fig. 15 shows the photograph of the finished compact filtering antenna.

\section{CONCLUSION}

A new printed filtering antenna with a very compact configuration has been demonstrated. By incorporating a ground-intruded miniaturized coupled line resonator, the proposed filtering antenna occupied about the same substrate area as a conventional printed antenna, while exhibited flat high band-edge selectivity, and good stop-band suppression. Thorough analysis and design of the coupled line resonator and the filtering antenna have been described. The measured results, including the return loss, total radiation power, and antenna gains versus frequency, have good agreement with the design ones.

\section{REFERENCES}

[1] F. Queudet, I. Pele, B. Froppier, Y. Mahe, and S. Toutain, "Integration of pass-band filters in patch antennas," in Proc. 32th Eur. Microw. Conf., 2002, pp. 685-688.

[2] J.-H. Lee, N. Kidera, S. Pinel, J. Laskar, and M. M. Tentzeris, "Fully integrated passive front-end solutions for a V-band LTCC wireless system," Antennas Wireless Propag. Lett., vol. 6, pp. 285-288, 2007.

[3] N. Yang, C. Caloz, and K. Wu, "Co-designed CPS UWB filter-antenna system," in Proc. IEEE AP-S Int. Symp., Jun. 2007, pp. 1433-1436.

[4] C.-H. Wu, C.-H. Wang, S.-Y. Chen, and C. H. Chen, "Balanced-tounbalanced bandpass filters and the antenna applications," IEEE Trans. Microw. Theory Tech., vol. 56, no. 11, pp. 2474-2482, Nov. 2008.

[5] H. Blondeaux, D. Baillargeat, P. Leveque, S. Verdeyme, P. Vaudon, P. Guillon, A. Carlier, and Y. Cailloce, "Microwave device combining and radiating functions for telecommunication satellites," in IEEE MTT-S Int. Microw. Symp. Dig., May 2001, pp. 137-140.
[6] T. L. Nadan, J. P. Coupez, S. Toutain, and C. Person, "Optimization and miniaturization of a filter/antenna multi-function module using a composite ceramic-foam substrate," in IEEE MTT-S Int. Microw. Symp. Dig., Jun. 1999, pp. 219-222.

[7] A. Abbaspour-Tamijani, J. Rizk, and G. Rebeiz, " Integration of filters and microstrip antennas," in Proc. IEEE AP-S Int. Symp., Jun. 2002, pp. $874-877$.

[8] S. Oda, S. Sakaguchi, H. Kanaya, R. K. Pokharel, and K. Yoshida, "Electrically small superconducting antennas with bandpass filters," IEEE Trans. Appl. Supercond., vol. 17, no. 2, pp. 878-881, Jun. 2007.

[9] C. Quendo, E. Rius, and C. Person, "Narrow bandpass filters using dual-behaviors resonators," IEEE Trans. Microw. Theory Tech., vol. 51, no. 3, pp. 734-743, Mar. 2003

[10] Z. Ma and Y. Kobayashi, "Design and realization of bandpass filters using composite resonators to obtain transmission zeros," in Proc. 35th Eur. Microw. Conf., 2005.

[11] C.-T. Chuang and S.-J. Chuang, "New printed filtering antenna with selectivity enhancement," in Proc. 39th Eur. Microw. Conf., 2009, pp. 747-750.

[12] N. Yildirim et al., "A revision of cascade synthesis theory covering cross-coupled filters," IEEE Trans. Microw. Theory Tech., vol. 50, no. 6, pp. 1536-1543, Jun. 2002

[13] D. M. Pozar, Microwave Engineering, 3nd ed. New York: Wiley, 2005 , ch. 8

[14] AWR Microwave Office (MWO). AWR Corporation. Segundo, CA, 2010.

[15] W. L. Stutzman and G. A. Thiele, Antenna Theory and Design. New York: Wiley, 1998.

[16] High Frequency Structure Simulator (HFSS). Ansoft Corporation. Pittsburgh, PA, 2001

[17] G. L. Mattaei, L. Young, and E. M. T. Jones, Microwave Filters, Impedance-Matching Network, and Coupling Structure. Norwood, MA: Artech House, 1980.

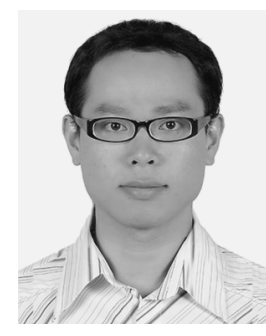

Chao-Tang Chuang was born in Hualien, Taiwan, R.O.C., on June 2, 1981. He received the B.S. degree in physics from the National Chung Hsing University, Taichung, Taiwan, in 2004 and the M.S. degree in electrical engineering from National Central University, Jungli, Taiwan, in 2006. He is currently working toward the Ph.D. degree in communication engineering in National Chiao Tung University, Hsinchu, Taiwan, R.O.C.

He current research interests include design of microwave circuits and antennas.

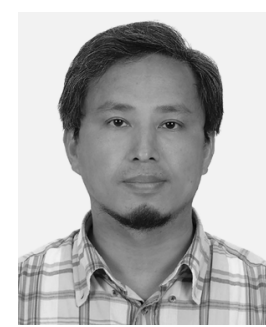

Shyh-Jong Chung (M'92-SM'06) was born in Taipei, Taiwan, R.O.C. He received the B.S.E.E. and $\mathrm{Ph} . \mathrm{D}$. degrees from National Taiwan University, Taipei, Taiwan, R.O.C., in 1984 and 1988, respectively.

Since 1988, he has been with the Department of Communication Engineering, National Chiao Tung University, Hsinchu, Taiwan, R.O.C., where he is currently a Professor and serves as the Director of the Institute of Communication Engineering. From September 1995 to August 1996, he was a Visiting Scholar with the Department of Electrical Engineering, Texas, A\&M University, College Station. His areas of interest include the design and applications of active and passive planar antennas, LTCC-based RF components and modules, packaging effects of microwave circuits, vehicle collision warning radars, and communications in intelligent transportation systems (ITSs).

Dr. Chung received the Outstanding Electrical Engineering Professor Award of the Chinese Institute of Electrical Engineering and the Teaching Excellence Awards of National Chiao Tung University both at 2005. He served as the Treasurer of IEEE Taipei Section from 2001 to 2003 and the Chairman of IEEE MTT-S Taipei Chapter from 2005 to 2007. 